EESTI NSV TEADUSTE AKADEEMIA TOIMETISED. XI KOIDE FOOSIKALIS-MATEMAATILISTE JA TEHNILISTE TEADUSTE SEERIA, 1962, NR. 1

ИЗВЕСТИЯ АКАДЕМИИ НАУК ЭСТОНСКОИ ССР. ТОМ ХI СЕРИЯ ФИЗИКО-МАТЕМАТИЧЕСКИХ Н ТЕХНИЧЕСКИХ НАУК. 1962, Ni 1

\title{
ОБ ОПТИЧЕСКИХ ЯВЛЕНИЯХ ПРИ ПРОСВЕЧИВАНИИ СТОПЫ ДВУПРЕЛОМЛЯЮЩИХ ПЛАСТИНОК
}

\author{
Х. АБЕН, \\ кандидат технических наук
}

\section{1. Введение}

В оптике анизотропных сред представляет большой интерес задача, при которой среда оптически неоднородна, т. е. когда эллипсоид Френеля изменяется в направлении распространения света,

Эта задата актуальна в кристаллооптике при анализе оптических явлений при просвечивании стопы двупреломляющих пластинок, С другой стороны, она представляет интерес в фотоупругости в случае применения метода сквозного просвечивания *

Для применения метода сквозного просвечивания необходимо выяснить, какие олтические величины можно экспериментально определить и каким образом эти величины связаны с напряженным состоянием модели. В работе ['] было доказано, что при просвечивании оптически анизотропного и неоднородного тела всегда существуют два перпендикулярных друг другу положения поляризатора, при которых выходящий нз тела свет линейно поляризован. При этом соотвегствующие направления колебания выходяцего света также перпендикулярны между собой. Эти направления называются х арактеристическим и направлениями. В работе ['] показано также, что из-за некоторых особых свойств характеристические направления играют исключительно важную роль при анализе оптических явлений при просвечивании анизотропных неоднородных сред.

На основе аналогии между оптически анизотропной и неоднородной средой и стопой двупреломляющих пластинок естественно предполагать, что характеристические направления существуют и в последнем случае. Как известно, задаче о стопе двупреломляющих пластинок посвящено много исследований. В общем виде эта задача впервые была рассмотрена Пуанкаре [2]. В работах Джонса и Хурвица [3-6] для анализа указанной задачи разработан эффективный метод с применением теории матриц. Некоторые результаты, полученные Пуанкаре, Джонсом и Хурвицем, а также Ричарцем и Хсиен Ю-хсю [7], позволяют предполагать существование характеристических направлений и в случае стопы двупреломляющих пластинок. Однако подробного рассмотрения этот вопрос пока не нашел.

Целью настоящей работы является разработка теории характеристических направлений для случая просвечивания стопы двупреломляющих пластинок.

В.следующих работах автора будет дано обобщение полученных результатов и рассмотрено применение разработанной теории при решении некоторых задач кристаллооптики.

- Методом сквозного просвечивания называется метод, при котором о напряжениях в сложнонапряженной модели судят по оптическим явлениям, возникающим при сквозном просвечиванин всей модели. 


\section{2. Просвечивание двух двупреломляющих пластинок}

Рассмотрим просвечивание двух поставленных друг за другом двупреломляющих пластинок. Обозначаем главные направления первой пластинки (I) $x_{1}, y_{1}$, второй пластинки (II) $x_{2}, y_{2}$, разности фаз соответственно $\Delta_{1}$ и $\Delta_{2} ; \beta$ - угол между соответствующими главными направлениями первой и второй пластинки (фиг. 1).

Пусть на первую пластинку падает линейно поляризованный свет с амплитудой 1 , направление колебания которого составляет с осью $x$ угол $\alpha_{1}$. Обозначаем одним штрихом световые колебания при входе в двупреломляющую пластинку и двумя штрихами - при выходе. Световое колебание при выходе из первой пластинки выражается уравнениями

$$
\begin{aligned}
& x_{1}^{\prime \prime}=\cos \alpha_{1} \sin \omega t, \\
& y_{1}^{\prime \prime}=\sin \alpha_{1} \sin \left(\omega t-\Delta_{1}\right) .
\end{aligned}
$$

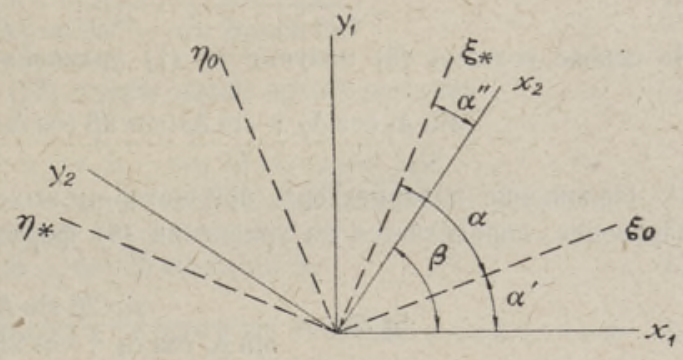

Фиг. 1.

Эллиптическое колебание (1) падает на вторую пластинку. Поэтому целесообразно привести световой эллипс (1) к главным направлениям второй пластинки. После преобразования координат имеем

$$
\begin{aligned}
& x_{2}^{\prime}=\left(\cos ^{2} \alpha_{1} \cos ^{2} \beta+\sin ^{2} \alpha_{1} \sin ^{2} \beta+\frac{1}{2} \sin 2 \alpha_{1} \sin 2 \beta \cos \Delta_{1}\right)^{1 / 2} \sin \left(\omega t-w_{1}\right), \\
& y_{2}^{\prime}=\left(\cos ^{2} \alpha_{1} \sin ^{2} \beta+\sin ^{2} \alpha_{1} \cos ^{2} \beta-\frac{1}{2} \sin 2 \alpha_{1} \sin 2 \beta \cos \Delta_{1}\right)^{1 / 2} \sin \left(\omega t-w_{2}\right),
\end{aligned}
$$

где

$$
\begin{aligned}
& \operatorname{tg} w_{1}=\frac{\sin \Delta_{1}}{\operatorname{ctg} \alpha_{1} \operatorname{ctg} \beta+\cos \Delta_{1}}, \\
& \operatorname{tg} w_{2}=\frac{\sin \Delta_{1}}{-\operatorname{ctg} \alpha_{1} \operatorname{tg} \beta+\cos \Delta_{1}} .
\end{aligned}
$$

Из выражений (2) получим для отношения амплитуд колебаний $y_{2}^{\prime}$ и $x_{2}^{\prime \prime}$, которое обозначаем ** $\operatorname{tg} \alpha_{2}$, следующее уравнение:

$$
\cos 2 \alpha_{2}=\cos 2 \alpha_{1} \cos 2 \beta+\sin 2 \alpha_{1} \sin 2 \beta \cos \Delta_{1} .
$$

Разность фаз, характеризующая выходящий из первой пластинки световой эллипс относительно главных направлений второй пластинки, выражается соотношением

$$
\operatorname{tg} \Delta_{2}^{\prime}=\operatorname{tg}\left(w_{2}-w_{1}\right)=\frac{\sin \Delta_{1}}{\cos \Delta_{1} \cos 2 \beta-\operatorname{ctg} 2 \alpha_{1} \sin 2 \beta} .
$$

Формула (5) впервые выведена Сенармоном [8].

Разность фаз световых колебаний при выходе из второй пластинки составляет

$$
\Delta_{2}^{\prime \prime}=\Delta_{2}^{\prime}+\Delta_{2}
$$

-. Положительное направление отсчета углов - протнв вращения часовой стрелкн.

-. Как известно, отношение амплитуд колебаний $y_{2}^{\prime \prime}$ и $x_{2}^{\prime \prime}$ характеризуется тем же углом $a_{2}$. 
На основе соотношений (5) и (6) имеем

$$
\operatorname{tg} \Delta_{2}^{\prime \prime}=\frac{\sin \Delta_{1} \cos \Delta_{2}+\sin \Delta_{2}\left(\cos 2 \beta \cos \Delta_{1}-\operatorname{ctg} 2 \alpha_{1} \sin 2 \beta\right)}{-\sin \Delta_{1} \sin \Delta_{2}+\cos \Delta_{2}\left(\cos 2 \beta \cos \Delta_{1}-\operatorname{ctg} 2 \alpha_{1} \sin 2 \beta\right)} .
$$

Для того, чтобы из второй пластинки выходил линейно поляризованный свет, должно быть выполнено условие

$$
\Delta_{2}^{\prime \prime}=m \pi, \quad(m=0,1,2 \ldots) .
$$

На основе условия (8) получим из (7) уравнение

$$
\sin \Delta_{1} \cos \Delta_{2}+\sin \Delta_{2}\left(\cos 2 \beta \cos \Delta_{1}-\operatorname{ctg} 2 \alpha_{1} \sin 2 \beta\right)=0 .
$$

Положение поляризатора, при котором выходящий из системы свет линей:о поляризован, определяется из уравнения (9) формулой

$$
\operatorname{tg} 2 \alpha^{\prime}=\frac{\sin 2 \beta \sin \Delta_{2}}{\sin \Delta_{1} \cos \Delta_{2}+\cos 2 \beta \cos \Delta_{1} \sin \Delta_{2}}
$$

где $\alpha_{1}$ в случае $\Delta_{2}^{\prime \prime}=m \pi$ обозначено через $\alpha^{\prime}$.

Из формулы (10) видно, что угол $\alpha^{\prime}$ имеет действительные значения при произвольных значениях величин $\Delta_{1}, \Delta_{2}$ и $\beta$. Следовательно можно заключить, что при просвечивании двух двупреломляющих пластинок всегда существуют два перпендикулярные друг другу положения поляризатора, при которых выходящий из второй пластинки свет линейно поляризован. Так как разности фаз $\Delta_{1}$ и $\Delta_{2}$ определены только при определенной длине волны, то сказанное справедливо в случае монохроматнческого света.

Чтобы найти направления колебания линейно поляризованного света, выходящего яз второй пластинки, подставим в соотношение (4) вместо $\alpha_{1}$ выражение $\boldsymbol{a}^{\prime}$ по формуле (10). После преобразований находим, обозначив $a_{2}$ в случае $\alpha_{1}=\alpha^{\prime}$ через $\alpha^{\prime \prime}$.

$$
\operatorname{tg} 2 \alpha^{\prime \prime}=\frac{ \pm \sin 2 \beta \sin \Delta_{1}}{\cos \Delta_{1} \sin \Delta_{2}+\cos 2 \beta \sin \Delta_{1} \cos \Delta_{2}} .
$$

Напоминаем, что угол $\alpha^{\prime \prime}$ относится к главным направлениям второй пластинки.

Рассмотрим теперь просвечивание нашей системы в противоположном направлении. Аналогично предыдущему можно доказать, что если поляризатор составляет с главным направлением пластинки II угол $\alpha^{\prime \prime}$, который определяется по формуле

$$
\operatorname{tg} 2 \alpha^{\prime \prime}=\frac{-\sin 2 \beta \sin \Delta_{1}}{\cos \Delta_{1} \sin \Delta_{2}+\cos 2 \beta \sin \Delta_{1} \cos \Delta_{2}}
$$

то из пластинки I выходит линейно поляризованный свет, направление колебания которого определяется формулой (10). Следовательно, в формуле (11) приходится в числителе учитывать знак минус.

Направления, определенные формулами (10) и (12), называем характеристическими направлениями рассматриваемой оптической системы. Характернстические направления в точке входа света $\left(\xi_{0}, \eta_{0}\right)$ называем перв ичными характеристическими направлениями, в точке выхода света $\left(\xi_{*}, \eta_{*}\right)-$ в тор и чными характеристическими направлениями (фиг. 1). Характеристические направления в точке входа и выхода света, которые могут одновременно совпадать с направлением колебания света, называем. сопря женны м характеристическими направлениями. Сопряженными являются характеристические направления $\xi_{0}$ и $\xi_{*}$, если при направлении колебания входящего света $\xi_{0}$ направление колебания выходящего из системы света сов-. падает с $\xi_{*}$. Сспряженными являются также характеристические направления $\eta_{0}$ и $\eta^{*}$ 
Как было показано, характеристические направления сохраняют свои свойства также при просвечивании системы в противоположном направлении. Следовательно, если на пластинку II падает линейно поляризованный свет с колебаниями в направлении $\xi_{*}\left(\eta_{*}\right)$, то из пластинки I выходит линейно поляризованный свет с колеба ниями в направленни $\xi_{0}\left(\eta_{0}\right)$.

Определяем разность фаз $\Delta_{\text {* }}$ между световыми колебаниями на вторичных характеристических направлениях $\xi_{\text {* и }} \eta_{*}$. Применяя формулу преобразования (5), получим

$$
\operatorname{tg} \Delta_{*}=\frac{\sin \Delta_{2}^{\prime \prime}}{\cos \Delta_{2}^{\prime \prime} \cos 2 \alpha^{\prime \prime}-\operatorname{ctg} 2 \alpha_{2} \sin 2 \alpha^{\prime \prime}} .
$$

На основе формул (4), (7), (12) и (13) имеем после преобразований

$$
\operatorname{tg} \Delta_{*}=\frac{\sqrt{1-\left(\cos \Delta_{1} \cos \Delta_{2}-\cos 2 \beta \sin \Delta_{1} \sin \Delta_{2}\right)^{2}}}{\cos \Delta_{1} \cos \Delta_{2}-\cos 2 \beta \sin \Delta_{1} \sin \Delta_{2}}
$$

или

$$
\cos \Delta_{*}=\cos \Delta_{1} \cos \Delta_{2}-\cos 2 \beta \sin \Delta_{1} \sin \Delta_{2} \text {. }
$$

Называем разность фаз $\Delta_{*}$ х арактеристической разностью фаз. Замечательно, что последняя является инвариантной относительно положения поляризатора, а также относительно направления просвечивания системы.

Отметим, что формулы, эквивалентные по содержанию формулам (10), (12) и (14), получены Ричарцем и Хсиен Ю-хсю[ $\left.{ }^{7}\right]$ путем применения теории матриц и алгебры кватернионов.

Докажем еще одно важное свойство характеристических направлений. Обозначаем отношение амплитуд колебаний на первичных характеристических направлениях $\operatorname{tg} \alpha_{0}$ и на вторнчных характеристических направлениях $\operatorname{tg} \alpha_{*}$. Будем искать зависимость угла $a_{*}$ от угла $a_{0}$.

В точке выхода света имеем

$$
\sin 2 \alpha_{2} \sin \Delta_{2}^{\prime \prime}=\sin 2 \alpha_{*} \sin \Delta_{*},
$$

так как величина $\sin 2 \alpha_{2} \sin \Delta_{2}^{\prime \prime}$ является инвариантной относительно преобразования координат (см. [9]). На основе соотношений (4), (7) и (15) получим из (16)

$$
\sin 2 \alpha_{*}=\sin 2\left(\alpha_{1}-a^{\prime}\right)
$$

и далее, учитывая, что $\alpha_{1}-\alpha^{\prime}=\alpha_{0}$,

$$
\sin 2 \alpha_{*}=\sin 2 \alpha_{0} .
$$

Равенство (18) показывает, что отношение амплитуд световых колебаннй на первичных и вторичных характеристических направлениях совпадает. Следовательно, на сопряженных характеристических направлениях амплитуды светового колебания равны между собой. Отметим, что это следует также из закона сохранения энергии светового колебания.

Выводим формулу для угла $\alpha$ между сопряженными характеристическими направлениями. По фиг. 1 имеем

$$
\alpha=\beta+\alpha^{\prime \prime}-\alpha^{\prime}
$$

На основе соотношений $(10)$, (12) и (19) получим

$$
\operatorname{tg} 2 \alpha=\frac{A}{B} \text {, }
$$

где

$$
\begin{aligned}
& A=\sin 2 \beta\left[\cos 2 \beta\left(1-\cos \Delta_{1}-\cos \Delta_{2}+\cos \Delta_{1} \cos \Delta_{2}\right)-\sin \Delta_{1} \sin \Delta_{2}\right] \\
& B=\cos ^{2} 2 \beta\left(1+\cos \Delta_{1} \cos \Delta_{2}\right)-\cos 2 \beta \sin \Delta_{1} \sin \Delta_{2}+\sin ^{2} 2 \beta\left(\cos \Delta_{1}+\cos \Delta_{2}\right) .
\end{aligned}
$$


Из изложенного следует, что характеристические направления и характеристическая разность фаз характеризуют систему из двух двупреломляющих пластинок аналогично главным направлениям и разности фаз. в случае одной двупреломляющей пластинки. Разница только в том, что главные направления системы - характеристические направления - в точке входа и выхода света составляют некоторый угол $\alpha$. Хотя физически более обоснованным может казаться описывание выходящего из системы светового колебания на главных направлениях второй пластинки, эти направления лишены свойств характеристических направлений. Кроме того, характеристические направления можно определить экспериментально при помощи полярископа с независимо поворачиваемыми поляризатором и анализатором, в то время как главные направления первой или второй пластинки экспериментально определить нельзя. Так же можно измерить характеристическую разность фаз $\Delta_{*}$, тогда как разность фаз $\Delta_{2}^{\prime \prime}$ экспериментально измерить невозможно.

Для измерения характеристической разности фаз можно применять как метод Сенармона, так и компенсатор. Методика измерения аналогична обычной. Приходится только иметь в виду, что равенство амплитуд на вторичных характеристических направлениях осуществляется положением поляризатора под углом $45^{\circ}$ относительно первичных характеристических направлений.

$$
\text { Если } \beta=0 \text {, то из (10) и (12) имеем }
$$

$$
\alpha^{\prime}=\alpha^{\prime \prime}=m \frac{\pi}{2}
$$

В данном случае сопряженные характеристические направления коллинеарны. Из (15) имеем

$$
\Delta_{*}=\Delta_{1}+\Delta_{2}
$$

Если $\beta=90^{\circ}$, то

$$
\Delta_{*}=\Delta_{1}-\Delta_{2}
$$

Эти результаты нзвестны из кристаллооптики.

\section{3. Просвечивание трех двупреломляющих пластинок}

Из предыдущего следует, что систему из двух двупреломляющих пластинок можно рассматривать как одну «сложную» двупреломляющую пластинку. Разностью фаз «сложной» пластинки является характеристическая разность фаз системы, главными направлениями в точке входа света - первичные характеристические направления и в точке выхода света - вторичные характеристические направления. Так, например, углом между направлением колебания падающего света и главными направлениями есложной» пластинки приходится считать угол между поляризатором и первичными характеристическими направлениями, а углом между главными направлениями «сложной» лластинки и третьей двупреломляющей пластинки - угол между вторичными характеристическими направлениями и главными направлениями третьей пластинки.

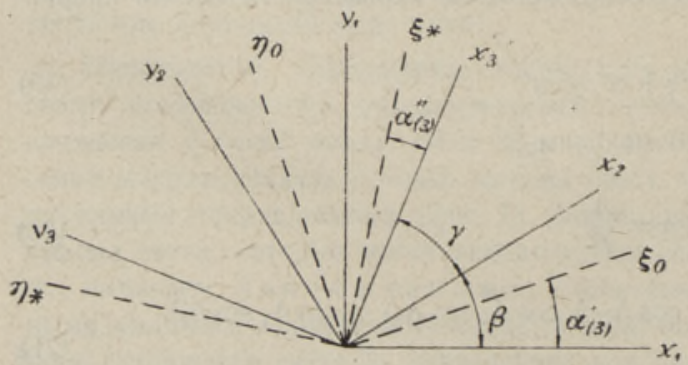

Фиг. 2.

Рассмотрим просвечивание трех двупреломляющих пластинок. Угол между главными направлениями второй и третьей пластинки обозначаем $\gamma$ (фиг. 2). Трактуем систему из первой и второй пластинки как одну ๔сложнуюж пластинку. Очевидно, что для системы из «сложнойэ пластинки и третьей пластинки действительны все положения предыдущей главы. На основе формул (10) и (12) можем 
выписать формулы для определения характеристических направлений системы из трех двупреломляющих пластинок

$$
\begin{aligned}
\operatorname{tg} 2 \bar{\alpha}_{(3)}^{\prime} & =\frac{\sin 2\left(\gamma-\alpha^{\prime \prime}\right) \sin \Delta_{3}}{\sin \Delta_{*} \cos \Delta_{3}+\cos 2\left(\gamma-\alpha^{\prime \prime}\right) \cos \Delta_{*} \sin \Delta_{3}}, \\
\operatorname{tg} 2 \alpha_{(3)}^{\prime \prime} & =\frac{-\sin 2\left(\gamma-\alpha^{\prime \prime}\right) \sin \Delta_{*}}{\cos \Delta_{*} \sin \Delta_{3}+\cos 2\left(\gamma-\alpha^{\prime \prime}\right) \sin \Delta_{*} \cos \Delta_{3}},
\end{aligned}
$$

где $\overline{\boldsymbol{a}}_{(3)}^{\prime}=\alpha_{(3)}^{\prime}-\boldsymbol{\alpha}^{\prime}$, причем $\boldsymbol{\alpha}_{(3)}^{\prime}$ есть угол между $x_{1}$ и первичным характеристическим направлением $\xi_{0}$ системы из трех пластинок, а $\alpha_{(3)}^{\prime \prime}$ - угол между $x_{3}$ и вторичным ха-

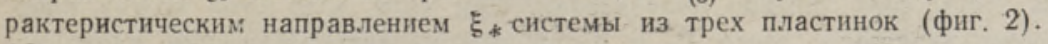

На основе соотношений $(10),(12),(15),(22)$ и (23) получим после преобразованнй

$$
\begin{aligned}
& \operatorname{tg} 2 \alpha_{(3)}^{\prime}=\frac{C}{D}, \\
& \operatorname{tg} 2 \alpha_{(3)}^{\prime \prime}=\frac{E}{F},
\end{aligned}
$$

где

$C=\sin 2 \beta \sin \Delta_{2} \cos \Delta_{3}+\sin 2 \beta \cos 2 \gamma \cos \Delta_{2} \sin \Delta_{3}+\cos 2 \beta \sin 2 \gamma \sin \Delta_{3}$,

$D=\sin \Delta_{1} \cos \Delta_{2} \cos \Delta_{3}+\cos 2 \beta \cos \Delta_{1}\left(\sin \Delta_{2} \cos \Delta_{3}+\cos 2 \gamma \cos \Delta_{2} \sin \Delta_{3}\right)-$

$$
-\sin \Delta_{3}\left(\sin 2 \beta \sin 2 \gamma \cos \Delta_{1}+\cos 2 \gamma \sin \Delta_{1} \sin \Delta_{2}\right),
$$

$E=-\sin 2 \gamma \cos \Delta_{1} \sin \Delta_{2}-\cos 2 \beta \sin 2 \gamma \sin \Delta_{1} \cos \Delta_{2}-\sin 2 \beta \cos 2 \gamma \sin \Delta_{1}$,

$F=\cos \Delta_{1} \cos \Delta_{2} \sin \Delta_{3}+\cos 2 \gamma \cos \Delta_{3}\left(\cos \Delta_{1} \sin \Delta_{2}+\cos 2 \beta \sin \Delta_{1} \cos \Delta_{2}\right)$

На основе формулы (15) имеем

$$
\cos \Delta_{*(3)}=\cos \Delta_{*} \cos \Delta_{3}-\cos 2\left(\gamma-\alpha^{\prime \prime}\right) \sin \Delta_{*} \sin \Delta_{3},
$$

откуда получим, учитывая соотношения (12) и (15),

$\cos \Delta_{*(3)}=\cos \Delta_{1} \cos \Delta_{2} \cos \Delta_{3}-\cos 2 \beta \sin \Delta_{1} \sin \Delta_{2} \cos \Delta_{3}-\cos 2 \gamma \cos \Delta_{1} \sin \Delta_{2} \sin \Delta_{3}-$ $-\cos 2 \beta \cos 2 \gamma \sin \Delta_{1} \cos \Delta_{2} \sin \Delta_{3}+\sin 2 \beta \sin 2 \gamma \sin \Delta_{1} \sin \Delta_{3}$.

Таким образом, система из трех двупреломляющих пластинок оптически характеризуется первичными и вторичными характеристическими направлениями и характеристической разностыю фаз, определяемыми формулами (24) - (31).

Отметим, что формулы $(10),(12),(15)$ и $(24)-(31)$ проверены экспериментально.

\section{4. Просвечивание стопы двупреломляющих пластинок}

Аналогично предыдущему можно рассматривать систему из трех двупреломляющих пластинок как одну єложную» двупреломляющую пластинку и определить характеристические направления и характеристическую разность фаз системы из четыpex двупреломляющих пластинок. Таким образом можно анализировать систему из произвольного числа двупреломляющих пластинок. При этом, очевидно, остаются в силе все свойства характеристических направлений и характеристической разности фаз, определенные во второй главе. Следовательно, изменение поляризации света при прохождении через стопу двупреломляющих пластинок полностью определяется характеристическими направлениями и характеристической разностью фаз. 
Отметим, что в работах Пуанкаре $\left[{ }^{2}\right]$ и Джонса и Хурвица $\left.{ }^{4}\right]$ доказано, что стопа двупреломляющих пластинок оптически эквивалентна одной двупреломляющей пластинке и повороту плоскостей поляризации. Однако, в указанных работах не рассмотрено физическое значение параметров эквивалентной оптической системы. По-видимому, под разностью фаз эквивалентной пластинки приходится подразумевать характеристическую разность фаз, а под углом поворота плоскостей поляризации угол $\alpha$ между сопряженными характеристическими направлениями.

Рассмотрим теперь некоторые частные случан.

Пусть характеристическап разность фаз стопы из $n$ двупреломляющих пластинок $\Delta_{*(n)}=2 m \pi(m=0,1,2 \ldots)$. В этом случае, независимо от положения поляризатора, выходящий из стопы свет линейно поляризован. При этом. если угол между плоскостью колебания входящего света $P_{0}$ и первичным характеристическим направлением $\xi_{0}$ равен $\alpha_{0}$, то угол между плоскостью колебания выходящего света $P$ * и вторичным характеристическим направлением $\xi_{*}$ также равен $\alpha_{0}$ (фиг. 3 ). Следо: вательно, в этом случае стопа двупреломляющих пластинок действует как ротатор. т. е., не изменяя световых колебаний, только поворачивает их на угол $\alpha$.

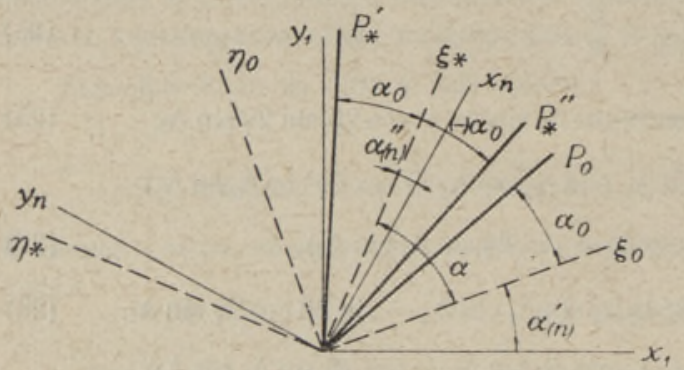

Фиг. 3.

Если $\Delta_{*(n)}=(2 m+1) \pi$, то при просвечивании стопы линейно поляризованным светом $P_{0}$ из стопы также выходит линейно поляризованный свет, плоскость колебания которого $P_{*}^{\prime \prime}$ составляет с $\xi_{*}$ угол $-\alpha_{0}$ (фиг. 3 ). Указанные явления следуют из свойств характеристических направлений.

Как известно, если разность фаз двупреломляющей пластинки равна $2 m \pi$, то невозможно определить ее главные направления, так как при любом положении поляризатора из пластинки выходит линейно поляризованный свет с неизменным направлением колебания. Аналогично, если характеристическая разность фаз стопы двупреломляющих пластинок равна $2 m \pi$, то нельзя определить ха рактеристические направления, так как при любом положении поляризатора из стопы выходит линейно поляризованный свет. Однако и в этом случае можно определить угол $\alpha$ между сопряженными характернстическими направлениями. В случае $\Delta_{*(\mathrm{n})}=$ $=(2 m+1) \pi$ также невозможно определить характеристические направления.

Отметим, что сопряженные характеристические направления стопы двупреломляющих пластинок могут быть и коллинеарны. При стопе из $n$ пластинок условие коллинеарности сопряженных характеристических направлений имеет вид

$$
\sum_{i=1}^{n-1} \beta_{i}=\alpha_{(n)}^{\prime}-\alpha_{(n)}^{\prime \prime},
$$

где $\beta_{i}-$ угол между главными направлениями пластинок $i$ и $i+1$.

Разумеется, при просвечивании стопы двупреломляющих пластинок монохроматическим светом различной длины волны возникают различные характеристические направления и характеристическая разность фаз. Это явление называется дисперсией характеристических величин.

\section{Л И Т Р А Т У Р А}

1. Х. К. Абен, Э. Г. С акс, Оптические явления при просвечивании оболочек. Поляризационно-оптический метод исследования напряжений (труды конференции), Изд. ЛГУ, 1960.

2. H. Poi n c a ré, Théorie mathématique de la lumière: II, Paris, 1892.

3. R. C. Jo nes, A new calculus for the treatment of optical systems I. J. Opt. Soc. Am., 31, 7, 1941. 
4. H. H urw itz, R. C. Jones, A new calculus for the treatment of optical systems II. J. Opt. Soc. Am., 31, 7, 1941.

5. R. C. J o n e s, A new calculus for the treatment of optical systems III. J. Opt. Soc. Am., $31,7,1941$.

6. R. C. Jon es, A new calculus for the treatment of optical systems IV. J. Opt. Soc. Am., 32, 8, 1942.

7. M. Richartz, Hsien-Yü Hs ü, Analysis of elliptical polarization. J. Opt. Soc. Am., 39, 2, 1949.

8. H. De $\mathrm{S}$ é n a rm on t, Sur les modifications que la réflexion spéculaire à la surface des corps métalliques imprime à un rayon de lumière polarisée. Ann. de chim. phys., (2), 73, 1840, p. 337.

9. F. E. W right, A spherical projection chart for use in the study of elliptically polarized light. J. Opt. Soc. Am., 20, 10, 1930.

Ннститут кибернетики

Академии наук Эстонской ССР
Поступила в редакцию

6. VII 1961

\title{
OPTILISTEST NĂHTUSTEST KAKSIKMURDVATE PLAATIDE PAKETI LÄBIVALGUSTAMISEL
}

\author{
H. Aben, \\ tehniliste teaduste kandidaat \\ Resümee
}

Artiklis näidatakse, et kaksikmurdvate plaatide paketi läbivalgustamisel esineb alati kaks teineteisega risti olevat polarisaatori asendit, mille puhul paketist väljuv valgus on lineaarselt polariseeritud. Seejuures on omavahel risti ka vastavad väljuva valguse vônkesuunad. Mainitud suundi nimetatakse optilise süsteemi karakteristlikeks suundadeks. Viimaste omadused on analoogilised peasuundade omadustele ühe kaksikmurdva plaadi puhul, kuid selle erinevusega, et sisenemispunkti ja väljumispunkti karakteristlikud suunad on teineteise suhtes teatud nurga võrra pööratud.

Tõestatakse, et faasinihe, mis iseloomustab optilisest süsteemist väljuva valguse ellipsit karakteristlike suundade suhtes, s. o. karakteristlik faasinihe, ei sõltu polarisaatori asendist. Karakteristlikud suunad ning karakteristlik faasinihe ei muutu süsteemi läbivalgustamisel vastassuunas ning nad määravad täielikult valguse polarisatsiooni muutuse kaksikmurdvate plaatide paketi läbivalgustamisel.

Eesti NSV Teaduste Akadeemia

Küberneetika Instituut
Saabus toimetusse

6. VII 1961

\section{ON THE OPTICAL PHENOMENA BY PASSING OF LIGHT THROUGH A PILE OF DOUBLE-REFRACTING PLATES}

\section{H. Aben}

\section{Summary}

It is shown that there exist always two perpendicular positions of polarizer by which the light emerging from a pile of double-refracting plates is linearly polarized. The corresponding directions of vibration of the emergent light are also perpendicular to each other. These directions are called the characteristic directions of the optical system. The properties of the characteristic directions are analogous to the properties of principal directions by a single double-refracting plate except that the characteristic directions at the point of incidence and at the point of emergence are rotated relatively to one another through an angle.

It is proved that the phase difference characterizing the ellipse of the emergent light relative to characteristic directions (the so-called characteristic phase difference) does not depend on the position of polarizer. The characteristic directions and characteristic phase difference do not change by passing of light-through the system in the opposite direction and they determine completely the change in polarization of the light by passing through a pile of double-refracting plates. 